Z. klin. Chem. u. klin. Biochem.

8. Jg., S. 278-283, Mai 1970

\title{
Untersuchungen des Elektrolytstoffwechsels der Erythrocyten bei chronischer Niereninsuffizienz
}

\author{
Von F. WESSELS \\ Aus der Mediqiniscben Universitäts-Poliklinik Münster/Westf. \\ (Direktor: Prof. Dr. H. Losse)
}

(Eingegangen am 5. Dezember 1969)

In Fortführung früherer Untersuchungen bestimmten wir bei Patienten mit chronischer Niereninsuffizienz verschiedener Schweregrade die Natrium- und Kaliumkonzentration in den Erythrocyten und im Plasma, den passiven Natriumtransport (Natriumeinstrom) der Erythrocyten sowie das aktuelle Blut-pH. Weiterhin prüften wir den Einfluß des Plasmas von Patienten mit chronischer Niereninsuffizienz auf den Natriumeinstrom und -gehalt der Erythrocyten von Normalpersonen. Wir kamen zu folgenden Ergebnissen:

Der Natriumeinstrom war bei Patienten mit einer chronischen Niereninsuffizienz aller Schweregrade verlangsamt. Eine Ausnahme bildeten die Patienten mit leichter bis mittelschwerer Niereninsuffizienz und Hochdruck. Bei diesen war der passive Natriumtransport leicht beschleunigt. Der Natriumgehalt der Erythrocyten war bei normotonen Patienten mit Niereninsuffizienz normal. Beim gleichzeitigen Bestehen einer Hypertonie war er leicht erhöht. Die Kaliumkonzentration der roten Blutkörperchen war unabhängig vom Bestehen eines Hochdrucks bei Patienten mit leichter bis mittelschwerer Niereninsuffizienz normal, bei schwerer Niereninsuffizienz herabgesetzt.

Zwischen dèm aktuellen Blut-pH und dem Natriumeinstrom sowie Natrium- und Kaliumgehalt der roten Blutkörperchen bestand eine signifikante Korrelation.

Plasma von Patienten mit chronischer Niereninsuffizienz hemmt auch an normalen Erythrocyten den Natriumeinstrom und bewirkt gleichzeitig eine Abnahme des Natriumgehaltes.

Die möglichen Ursachen der Elektrolytstoffwechselstörungen der Erythrocyten bei chronischer Niereninsuffizienz wie z. B. Azidose oder Hyperaldosteronismus werden diskutiert.

\section{Studies on the electrolyte metabolism of erytbrocytes in chronic kidney insufficiency}

In continuation of earlier studies, the concentrations of sodium and potassium in erythrocytes and plasma, the passive sodium transport (sodium influx) of the erythrocytes, and the actual blood $\mathrm{pH}$ were measured in patients with varying degrees of chronic kidney insuffciency. In addition, the effect of plasma from patients with chronic kidney insufficiency on the sodium influx and sodium content of erythrocytes from normal persons was tested. The following results were obtained: The sodium influx was decreased in cases of chronic kidney insufficiency of varying degrees of severity.

The patients with slight or intermediate kidney insufficiency accompanied by hypertension were an exception, in which the passive sodium transport was slightly accelerated. The sodium content of the exythrocytes was normal in patients with kidney insufficiency and normal blood pressure, but it was slightly elevated when the kidney insufficiency was accompanied by hypertension. In patients with a slight to intermediate kidney insufficiency the potassium concentration of the erythrocytes was normal and was not affected by hypertension, but it was decreased in severe kidney insufficiency.

There was a significant correlation between the actual blood $\mathrm{pH}$, the sodium influx, and the sodium and potassium content of the erythrocytes.

The plasma of patients with chronic kidney insufficiency also inhibits the sodium influx and causes a decrease in the sodium.content of normal erythrocytes. Possible causes of the disturbances of the electrolyte metabolism of the erythrocytes in chronic kidney insufficiency, e. g. acidosis or hyperaldosteronism are discussed.

Störungen des Elektrolyt- und Wasserhaushaltes sind häufige Komplikationen einer Niereninsuffizienz. Soweit diese Elektrolytverschiebungen den Extrazellulärraum betreffen, z. B. in Form einer Verminderung des Natrium-, Calcium und Chloridgehalts oder einer Steigerung der Kaliumkonzentration des Serums, sind sie schon lange bekannt. Erst in den letzten Jahren erlangte man vor allem durch die Analyse der menschlichen Erythrocyten auch Einblick in die intrazellulären Elektrolytverhältnisse bei der chronischen Niereninsuffizienz. Im allgemeinen wurde eine Verminderung der Kalium- und eine Steigerung der Natriumkonzentration gefunden (1-5).

Die intrazelluläre Natrium- und Kaliumkonzeñtration wird vor allem durch die Kationenpermeabilität und die Natrium-(Kalium-)pumpe reguliert.
Der aktive Kationentransport (Natrium-Kaliumpumpe) ist vom Energiestoffwechsel der Zelle abhängig. Durch Ultrafiltrate von Urämikerseren konnte in vitro eine Störung des Energiestoffwechsels verschiedener Gewebe einschließlich der Erythrocyten hervorgerufen werden $(6,7,3)$. Andererseits sprechen elektrophysiologische Untersuchungen von BoLte und Mitarbeitern an der Skelettmuskulatur für eine Steigerung der Membranpermeabilität und damit auch des passiven Kationentransports bei Patienten mit einer Niereninsuffizienz (8). ZurKlärung der Frage, inwieweit die Elektrolytverschiebungen in den Erythrocyten bei Patienten mit chronischer Niereninsuffizienz über eine Änderung des passiven oder des aktiven Kationentransports hervorgerufen werden, prüften wir den Natriumeinstrom sowie den Kaliumund Natriumgehalt der Erythrocyten dieser Patienten. 


\section{Krankengut und Methodik}

Gruppe I umfaßt 31 gesunde normotone Probanden ohne familiäre Hochdruckbelastung.

Gruppe II besteht aus 25 Patienten mit einer leichten bis mittelschweren chronischen Niereninsuffizienz (Blutharnstoff-N $<100 \mathrm{mg} / 100 \mathrm{ml}$ ), von denen 11 normoton waren (Gruppc II a), während 14 eine Hypertonie aufwiesen (Gruppe Il b). Von letzteren gaben 7 eine familiäre Hochdruckbelastung an. Patienten mit einer schweren chronischen Niereninsuffizienz (Blutharnstoff-N $>100 \mathrm{mg} / 100 \mathrm{ml}$ ) bzw. einer Urämie sind in der Gruppe III zusammengefaßt und zwar die normotonen $(n=11)$ in Gruppe III a und die hypertonen Patienten in Gruppe III b. 6 dieser Patienten waren familiär hochdruckbelastet. Der Natrium-Einstrom wurde nach der von Solomon (9) angegebenen Methode mit geringen, von uns früher bereits ausführlich beschriebenen Modifikationen bestimmt (10). Diese hatten wir jetzt weiter hinsichtlich der Bestimmung des Gesamtnatriumgehaltes sowie der frei austauschbaren Natriumfraktion $\left(q_{\infty}\right)$ folgendermaßen abgeändert: $3 \mathrm{~m} /$ des heparinisierten Vollbluts wurden $4 \mathrm{mal}$ in isotoner $\mathrm{MgCl}_{2}-$ Lösung gewaschen. Das so gewonnene plasmafreie Erythrocytenkonzentrat wurde mit bidest. Wasser bis auf $50 \mathrm{~m} /$ verdünnt und dadurch hämolysiert. Die Bestimmung der Natrium- und Kaliumkonzentration erfolgte mit dem Flammenphotometer (Eppendorf). Mit Hilfe des Hämatokritwertes wurde das Gesamtvolumen der in den $3 \mathrm{ml}$ enthaltenen Erythrocyten und mit Hilfe dieses Wertes die Natrium- und Kaliumkonzentration/Liter Erythrocyten bcrechnet. Der so erhaltene Wert für den gesamten intrazellulären Natriumgehalt entspricht wahrscheinlich, solange er nicht wesentlich erhöht ist, dem des frei austauschbaren Natriums $\left(q_{\infty}\right) . \mathrm{Zu}$ dieser Auffassung gelangten wir einmal durch Bestimmung des frei austauschbaren Natriums nach der von Sozomon angegebenen Methodik (9), bei der die Erythrocyten mit ${ }^{22} \mathrm{Na}$ über eine Dauer von 8-12 Stdn. inkubiert werden. Weiterhin sollten nach SozoMON die $\ln \left(1-\mathrm{q} / \mathrm{q}_{\infty}\right)$-Werte auf einer Geraden liegen, wenn man sie gegen die Zeit abträgt. Diese Forderung wurde praktisch regelmäßig auch dann erfüllt, wenn das gesamte intrazelluläre Natrium in die Funktion eingesetzt wurde, vorausgesetzt dieses betrug weniger als $8 \mathrm{mVal} / \mathrm{l}$ Erythrocyten. Erst bei einem höheren Gesamtnatrium der Erythrocyten fanden wir auch eine langsam austauschbare Natriumfraktion, die proportional zur Höhe des gesamten intrazellulären Natriumgehaltes anstieg. In diesen Fällen bestimmten wir daher auch weiterhin regelmäßig die frei austauschbare Natriumfraktion nach der Solomonschen Methode (9).

Die Bestimmung des aktuellen $\mathrm{pH}$ erfolgte in arteriell entnommenen Blutproben im Astrup-Mikroequilibriment der Firma Radiometer, Kopenhagen.

Weiterhin prüften wir den Einfluß des Plasmas von 8 Patienten mit einer Urämie auf den Natriumeinstrom und -gehalt der Erythrocyten von 3 Normalpersonen mit der Blutgruppe 0.

\section{Ergebnisse}

Wie die Abbildung 1 zeigt, ist der Na-Einstrom in die Erythrocyten bei Patienten mit chronischer Niereninsuffizienz verlangsamt. Eine Ausnahme bilden die

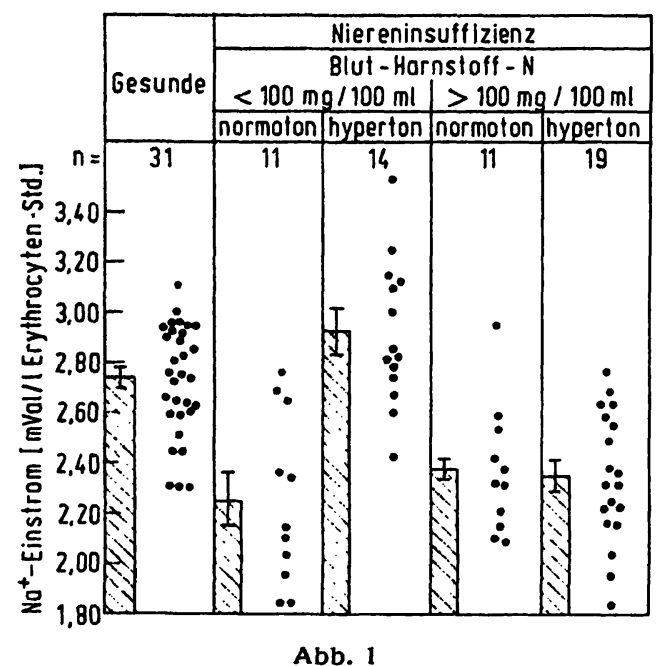

Natriumeinstrom in Erythrocyten bei Gesunden sowie Normo- und Hypertonikern mit Niereninsuffizienz

Patienten mit einer leichten bis mittelschweren renalen Insuffizienz und einem Hochdruck. Bei diesen findet sich eine leichte, jedoch signifikante Erhöhung des Natriumeinstroms.

Die Natriumkonzentration der Erythrocyten liegt, wie aus Abbildung 2a zu entnehmen ist, bei Patienten mit Niereninsuffizienz ohne Hochdruck im Normbereich, während sie bei niereninsuffizienten Hypertonikern signifikant erhöht ist.

Der Kaliumgehalt der Erythrocyten ist nach Abbildung $2 \mathrm{~b}$ unabhängig vom Vorliegen eines Hypertonus bei leichter bis mittelschwerer Niereninsuffizienz normal und bei schwerer Niereninsuffizienz vermindert. Die Mittelwerte mit Signifikanzberechnung sind in der Tabelle 1 zusammengefaßt.

Aus der Tabelle 1 läßt sich weiterhin ersehen, daß das aktuelle $\mathrm{pH}$ des Blutes bei leichter bis mittelschwerer Niereninsuffizienz im unteren Normbereich liegt, bei
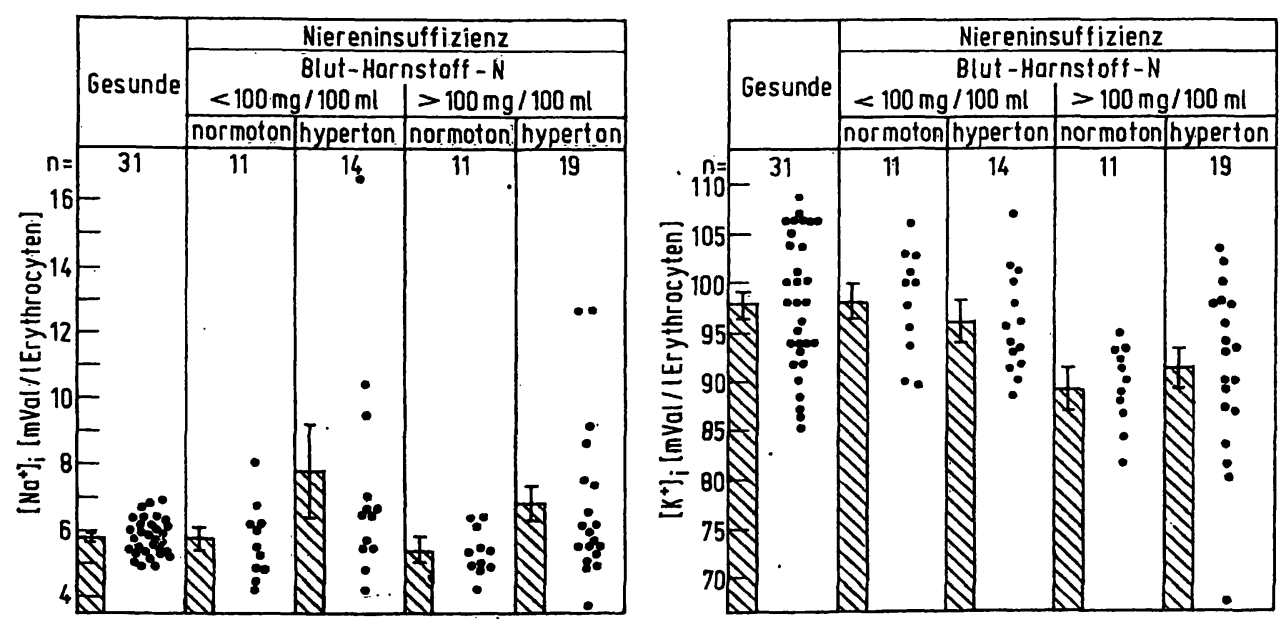

Abb. 2

Natrium- und Kaliumkonzentration der Erythrocyten bei Gesunden sowie bei Normo- und Hypertonikern mit Niereninsuffizienz 
Tab. 1

Natriumeinstrom in die Erythrocyten, Natrium- und Kaliumkonzentration der roten Blutkörperchen und des Plasmas sowie aktuelles pH bei Normalpersonen, Normo- und Hypertonikern mit Niereninsuffizienz

\begin{tabular}{|c|c|c|c|c|c|c|c|}
\hline \multirow[b]{2}{*}{ Gruppe } & \multirow{2}{*}{\multicolumn{2}{|c|}{ Kontroll-Personen }} & \multicolumn{5}{|c|}{ Niereninsuffizienz } \\
\hline & & & \multicolumn{2}{|c|}{$\begin{array}{l}\text { Blutharnstoff-N } \\
\text { Normoton. } \\
\quad \text { II a }\end{array}$} & $<100 \underset{\text { Hyperton. }}{\text { IIb }}$ & \multicolumn{2}{|c|}{$\begin{array}{c}\begin{array}{c}\text { Blutharnstoff-N }>100 \mathrm{mg} / 100 \mathrm{ml} \\
\text { Normoton. } \\
\text { III a }\end{array} \\
\text { Hyperton. } \\
\text { III b }\end{array}$} \\
\hline \multirow{2}{*}{$\begin{array}{l}\text { Anzahl } \\
\text { Na-Einstrom } \\
\text { (mVal/l Ery. Std.) } \\
{\left[\mathrm{Na}^{+}\right]_{1}(\mathrm{mVal} / l \text { Ery.) }}\end{array}$} & 2,73 & $\begin{array}{l}31 \\
\pm 0,0395\end{array}$ & \multirow{2}{*}{\multicolumn{2}{|c|}{$\begin{array}{l}11 \\
2,249 \pm 0,104 \\
P^{*}<0,001 \\
5,686 \pm 0,342 \\
* *\end{array}$}} & \multirow{2}{*}{$\begin{array}{l}2,14 \\
2,923 \pm 0,119 \\
* P<0,05 \\
7,783 \pm 1,42 \\
* \quad P<0,02\end{array}$} & \multirow{3}{*}{$\begin{aligned} & 11 \\
2,375 & \pm 0,422 \\
* & \geq 0,01 \\
5,375 & \pm 0,333 \\
* * & \\
89,166 & \pm 2,22 \\
* \quad P & <0,01\end{aligned}$} & \multirow{4}{*}{ 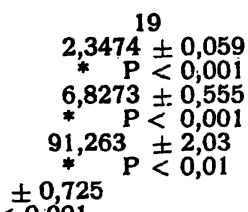 } \\
\hline & 5,819 & $\pm 0,094$ & & & & & \\
\hline$\left[\mathrm{K}^{+}\right]_{\mathrm{l}}$ (mVal/l Ery.) & 97,82 & $\pm 1,25$ & \multirow{4}{*}{$\underset{* *}{98,18}$} & $\pm 1,62$ & $96,0 \pm 2,01$ & & \\
\hline$\left[\mathrm{Na}^{+}\right]_{\mathrm{e}}$ (mVal $/ l$ Plasma) & 140,70 & $\pm 0,594$ & & \multirow{3}{*}{\multicolumn{2}{|c|}{$\begin{array}{rl}138,91 & \pm 0,743 \\
* * & \\
4,385 & \pm 0,129 \\
* & \mathbf{P} \\
7,375 & \pm 0,02 \\
7,0194\end{array}$}} & \multirow{3}{*}{\multicolumn{2}{|c|}{$\begin{array}{c}136,884 \pm 0,725 \\
\mathbf{P}<0,001 \\
5,081 \pm 0,183 \\
\mathbf{P}<0,001 \\
7,301 \pm 0,0494 \\
{ }_{*} \mathbf{P}<0,01\end{array}$}} \\
\hline$\left[\mathrm{K}^{+}\right]_{\mathrm{e}}$ (mVal/l Plasma) & 3,95 & $\pm 0,0627$ & & & & & \\
\hline $\mathrm{pH}$ & 7,40 & $\pm 0,04^{\circ}$ & & & & & \\
\hline
\end{tabular}

Mittelwerte $=\overline{\mathbf{x}} \pm \mathrm{s} \overline{\mathrm{x}} . \quad *$ Signifikanz gegen Gruppe 1. $\quad * *$ Keine Signifikanz gegen Gruppe I. $\quad 0^{\circ}=$ Normalwert des KlinikIabors $(\overline{\mathrm{x}} \pm 2 \mathrm{~s}$ ).

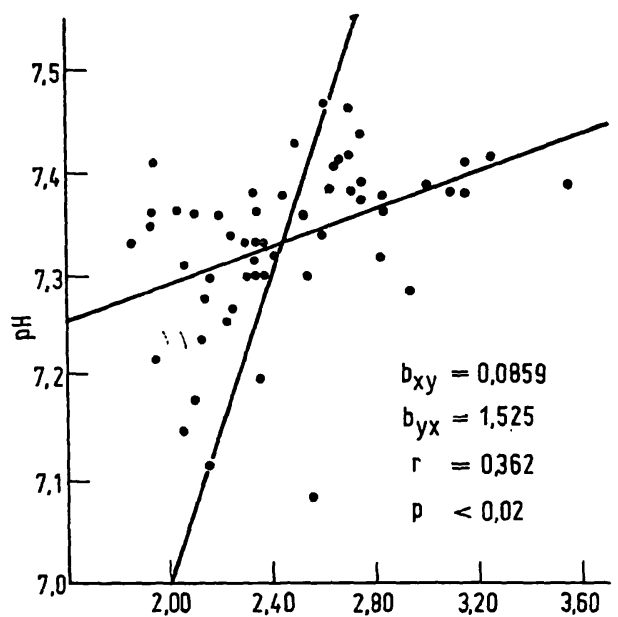

$\mathrm{Na}^{+}$- Einstrom ImVal /IErythrocyten. Std.]

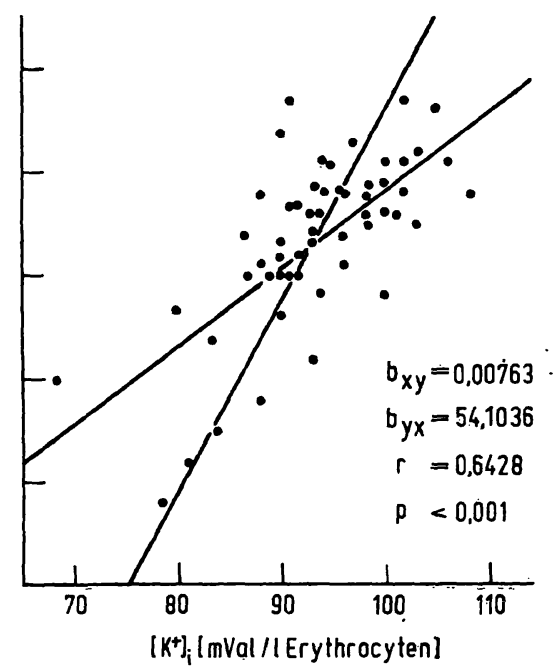

$\left[\mathrm{K}^{+}\right]_{\mathrm{i}}$ [mVal / Erythrocyten]

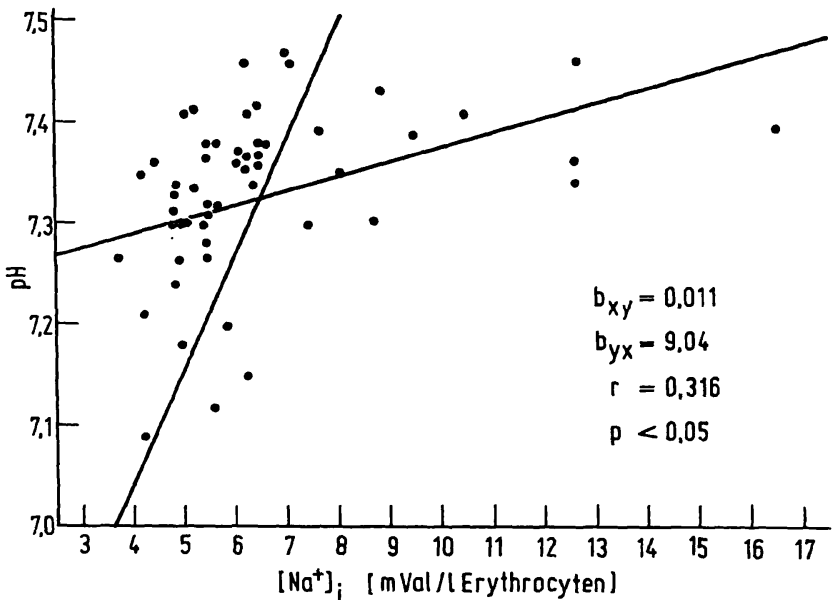

Abb. 4

Beziehung zwischen aktuellem Blut-pH und Natriumgehalt der Erythrocyten bei Patienten mit chronischer Niereninsuffizienz

schwerer Niereninsuffizienz dagegen deutlich erniedrigt ist.

Die Natriumkonzentration des Plasma ist nur bei schwerer Niereninsuffizienz leicht vermindert. Der Kaliumgehalt des $\mathrm{Plasmas}$ steigt mit zunehmender Niereninsuffizienz an.

Die Abbildungen $3 \mathrm{a}, 3 \mathrm{~b}$ und 4 demonstrieren, daß das aktuelle $\mathrm{pH}$ des Blutes leicht mit dem Natriumeinstrom und -gehalt, dagegen deutlich mit der Kaliumkonzentration korreliert ist.
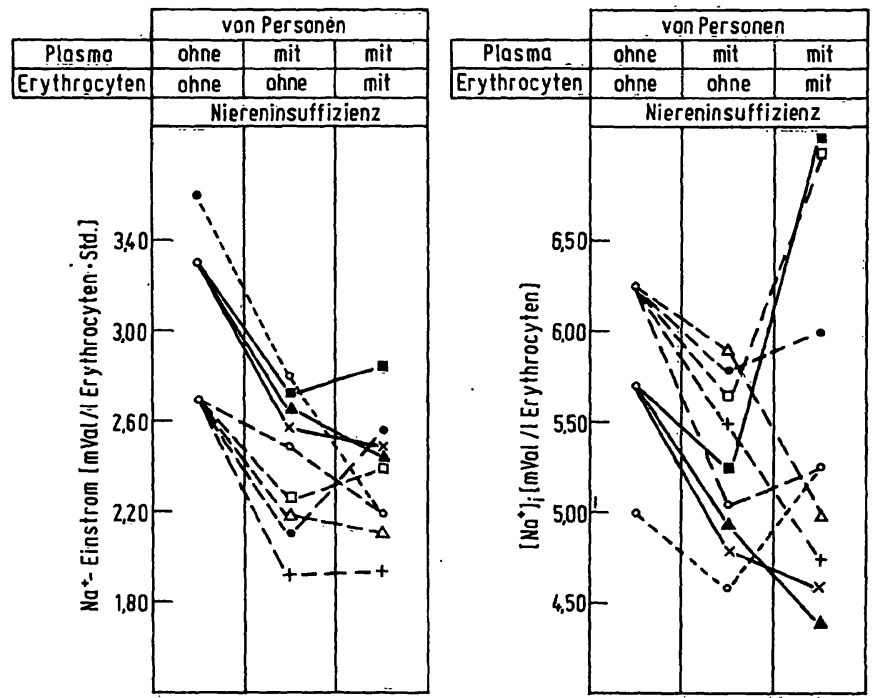

Abb. 5

Abb. 3

Beziehung zwischen aktuellem Blut-pH und Natriumeinstrom bzw. Kaliumgehalt der Erythrocyten bei Patienten mit chronischer Niereninsuffizien

Einfluß von Urämikerplasma auf Natriumeinstrom und -gehalt der Erythrocyten von Normalpersonen $\left.\begin{array}{cc}\text { Personen } & 0 \\ \text { mit Niereninsuffizienz } & 0 \\ 0 & 0\end{array}\right\}$ Hypertoniker $\left.\quad \stackrel{+}{x}\right\}$ Normotoniker

Wie Abbildung 5 zeigt, hemmt Plasma von Patienten mit Urämie auch den Natriumeinstrom in Erythrocyten von Normalpersonen. Gleichzeitig kommt es zu einer deutlichen Abnahme. der Natriumkonzentration der normalen Erythrocyten. 


\section{Diskussion}

Unsere Untersuchungen zeigen, daß bei Patienten mit chronischer Niereninsuffizienz der Natriumeinstrom in die Erythrocyten deutlich verlangsamt ist. Eine Ausnahme bilden lediglich die Patienten mit leichter bis mittelschwerer Niereninsuffizienz und Hochdruck (Gruppe IIb), bei denen der Natriuminflux leicht erhöht war.

Der passive Natrium- und Kaliumtransport durch die Erythrocytenmembran wird im wesentlichen durch den elektrochemischen Potentialgradienten der einzelnen Kationen und die Membranpermeabilität für diese Ionen bestimmt. Da das Konzentrationsgefälle zwischen Extra- und Intrazellulärraum für Natrium-Ionen bei dem Großteil unserer Patienten mit Niereninsuffizienz gegenüber Normalpersonen nicht oder nur leicht vermindert ist, müßte die Abnahme des Natriumeinstroms im wesentlichen auf einer Abnahme der Natriumpermeabilität beruhen.

Das gegenteilige Verhalten der Erythrocyten bei der Gruppe mit leichter bis mittelschwerer Niereninsuffizienz und Hypertonie läßt sich evtl. damit erklären, daß bei etwa 50\% dieser Patienten eine familiäre Hochdruckbelastung vorlag, bei der, wie wir in früheren Untersuchungen zeigen konnten, meistens der passive $\mathrm{Na}$ triumtransport beschleunigt ist (10).

Auf der Suche nach den Ursachen der Verminderung der Natriumpermeabilität konnten wir nachweisen, daß sich die Hemmung des Natriumeinstroms in die Erythrocyten bei Patienten mit Niereninsuffizienz mit dem Plasma auf Erythrocyten von Normalpersonen übertragen läßt. Sie ist also auf einen humoralen Faktor zurückzuführen, der, da er auch an kernlosen Erythrocyten wirksam ist, anscheinend direkt an der Zellmembran angreift und nicht, wie z. B. viele Hormone, eines Zellkerns bedarf, um den Natriumtransport beeinflussen zu können.

Weiterhin fanden wir bei Patienten mit chronischer Niereninsuffizienz eine signifikante Korrelation zwischen dem Natriumeinstrom in die Erythrocyten und dem aktuellen pH-Wert des Blutes. Bereits Solomon und Mitarbeiter (9) konnten an Menschenerythrocyten in vitro durch eine pH-Erniedrigung des Inkubationsmediums von 7,39 auf 6,94 eine Abnahme des Natriumeinstroms um durchschnittlich $23 \%$ erzielen. Pfleger und Mitarbeiter (11) wiesen an Kaninchenerythrocyten ebenfalls eine Abhängigkeit des passiven Natrium- und Kaliumtransports vom $\mathrm{pH}$ der Inkubationsflüssigkeit nach. Die Hemmung des Natriumeinstroms der Erythrocyten bei chronischer Niereninsuffizienz ließe sich damit einleuchtend durch die bei dieser Erkrankung häufig auftretende Azidose erklären. Andererseits war die Korrelation zwischen aktuellem $\mathrm{pH}$-Wert des Blutes und Natriumeinstrom verhältnismäßig gering $(r=$ 0,362 ), und außerdem fand sich bei einigen der niereninsuffizienten Patienten eine deutliche Verlangsamung des Natriumeinstroms, 'ohne daß eine wesentliche Azidose nachweisbar war. Auch bei den Ubertragungsversuchen sahen wir nur eine relativ lockere Beziehung zwischen dem $\mathrm{pH}-$ Wert des Plasmas von Patienten mit chronischer Niereninsuffizienz und dessen Hemmwirkung auf den Natriumeinstrom in die Erythrocyten von Normalpersonen. Deshalb möchten wir annehmen, daß außer der Azidose noch andere, bisher unbekannte Faktoren (sog. Urämiegifte?) für die Abnahme der Natriumpermeabilität der Erythrocyten mitverantwortlich sind.

$\mathrm{Da}$ die extra- und intrazelluläre Natriumkonzentration jm Untersuchungszeitraum weitgehend konstant blieb, müssen Natriumein- und -ausstrom gleich groß sein. Es besteht somit bei Niereninsuffizienz nicht nur eine Verlangsamung des Natriumeinstroms, sondern auch des Natriumausstroms, das heißt des aktiven Natriumtransports (Natriumpumpe). Eine Hemmung der Natriumpumpe der Erythrocyten bei Patienten mit chronischer Niereninsuffizienz wurde auch von VilLAMIL und Mitarbeitern (12) beobachtet. Die Autoren konnten diese Störungen des aktiven Natriumtransports durch Inkubation der Urämikererythrocyten in normalem Plasma nicht beseitigen und andererseits auch nicht mit dem Plasma von Urämikern oder durch Zugabe von Harnstoff zu normalem Plasma an normalen Erythrocyten hervorrufen.

Eine Hemmung des aktiven Kaliumtransports bei chronischer Niereninsuffizienz wurde von WELT und Mitarbeitern (3) beschrieben. Diese Beobachtung ist eine weitere Bestätigung unserer Befunde, da nach unseren heutigen Kenntnissen aktiver Natrium- und Kaliumtransport miteinander gekoppelt sind.

Andererseits wird der Natriumausstrom (Natriumpumpe) auch vom Substratangebot d. h. der intrazellulären Natriumkonzentration beeinflußt (13). Diese Tatsache ist für die meisten der von uns untersuchten Fälle ohne Bedeutung, da bei diesen der Natriumgehalt der Erythrocyten im Normbereich lag. Bei etwa 30\% unserer Patienten bestand jedoch eine mehr oder weniger starke Zunahme der Natriumkonzentration der Erythrocyten. In diesen Fällen muß man annehmen, daß die aus unseren Untersuchungen abzuleitende mäßige Verlangsamung des Natriumausstroms einer starken Hemmung der Natriumpumpe entspricht.

Plasma von Patienten mit Niereninsuffizienz bewirkt nach unseren Untersuchungen an normalen Erythrocyten außer der Hemmung des Natriumeinstroms auch eine Abnahme des Natriumgehalts. Das bedeutet, daß Urämikerplasma den Natriumausstrom (Natriumpumpe) erheblich weniger als den Natriumeinstrom oder auch entsprechend den Befunden Villamrls und Mitarbeitern gat nicht hemmt. Der aktive Natriumtransport der Erythrocyten ist ebenso wie der anderer Körperzellen abhängig vom Energiestoffwechsel. Dieser aber wird durch Urämikerseren bzw. durch Ultrafiltrate dieser Seren gestört $(6,7,14)$, anscheinend jedoch nicht so sehr, daß daraus eine wesentliche Hemmung der Natriumpumpe der roten Blutkörperchen resultiert (12). Die Verlangsamung des aktiven Natriumtransports ist nach den Befunden von WELT und Mitarbeitern wahrscheinlich auf einen herabgesetzten ATPase-Gehalt der Erythrocyten zurückzuführen (3). ATP als Energie- 
träger ist dagegen im Uberschuß vorhanden (3). Da die Erythrocyten nach dem Verlust ihres Kerns keine Enzyme mehr bilden können (15), wäre es zu erwägen, daß die sogenannten Urämiegifte bereits in den kernhaltigen Vorstufen der Erythrocyten die ATPase-Bildung stören. Der fehlende Einfluß von Urämikerplasma auf die Natriumpumpe von normalen Erythrocyten sowie von normalem Plasma auf den aktiven Natriumtransport von Urämikererythrocyten, ließe sich jedenfalls mit dieser Annahme einleuchtend erklären.

Eine Zunahme des Natriumgehalts der Erythrocyten fanden wir auffälligerweise nur bei einigen der niereninsuffizienten Patienten, die gleichzeitig einen Hypertonus aufwiesen. Sie war $z$. T. so stark, daß daraus eine leichte Steigerung der Natriumkonzentration der roten Blutkörperchen auch der gesamten Gruppe resultierte. Frühere Untersuchungen unserer Arbeitsgruppe $(4,5)$ sowie von GeSsLER (2) hatten bei schwerer Niereninsuffizienz (Blutharnstoff-N über $100 \mathrm{bzw} .120 \mathrm{mg} / 100 \mathrm{~m} /$ ) in einem höheren Prozentsatz der Patienten eine $\mathrm{Zu}-$ nahme des Natriumgehalts der Erythrocyten ergeben. Dieser Unterschied gegenüber unseren jetzigen Untersuchungen idürfte unseres Erachtens methodisch bedingt sein. Ebenso wie Gessler und Mitarbeiter (2) führten wir damals unsere Untersúchungen an einem nur durch Zentrifugieren gewonnenen Erythrocytensediment durch. Dieses Erythrocytensediment enthält Plasma, das durch seinen erheblich höheren Natriumgehalt den mit dieser Methode erhaltenen Wert der intrazellulären Natriumkonzentration fälschlich erhöht. Dieser sogenannte Plasmafehler ist normalerweise weitgehend konstant (um 5\%), wird jedoch bei starker Anämie (Hämatokrit unter 30\%) nach unseren in der letzten Zeit gewonnenen Erfahrungen z. T. deutlich größer. $\mathrm{Da}$ bei schwerer Niereninsuffizienz fast regelmäßig eine erhebliche Anämie besteht, wird bei Verwendung ungewaschener Erythrocyten allein schon durch den erhöhten Plasmafehler eine Zunahme des Natriumgehalts der Erythrocyten vorgetäuscht. Unsere jetzigen Ergebnisse stimmen im wesentlichen mit denen einiger anderer Untersucher überein, die entweder ebenfalls gewaschene Exythrocyten (3) verwandten oder den sogenannten Plasmafehler durch radioaktive Markierung des Plasmas maßen und damit ausschalten konnten $(1,12)$.

Da bei den Normotonikern mit schwerer Niereninsuffizienz die Natriumkonzentration der Erythrocyten normal ausfällt, kommt nach unseren Untersuchungen die Niereninsuffizienz selbst kaum als Ursache des intrazellulären Natriumanstiegs in Frage. Ein Zusammenhang mit der Hypertonie ist dagegen wahrscheinlicher. Dabei fällt auf, daß bei den meisten Patienten mit hohem intrazellulärem Natrium der Hochdruck sehr schwer, oft maligne war. Bekanntlich besteht bei schwerer oder maligner Hypertonie unabhängig von der Genese häufig ein sekundärer Hyperaldosteronismus (16). Da Aldosteron z. T. über die Nieren eliminiert wird, kann eine Niereninsuffizienz diesen Hyperaldosteronismus verstärken (17). KoCZOREK sah nach einer. längeren Behandlung mit Aldosteron einen Anstieg des Natriumgehaltes der Erythrocyten (18). Wir selbst fanden bei Oedemkranken, bei denen ja häufig ein sekundärer Hyperaldosteronismus besteht, ebenfalls eine deutlich exhöhte Natriumkonzentration in den roten Blutkörperchen (19). Im akuten Versuch konnten wir mit Aldosteron oder Desoxycorticosteronacetat eine Beschleunigung des passiven Natriumtransports der Erythrocyten hervorrufen (29).

Weiterhin führt ein Hyperaldosteronismus normalerweise zu einer Alkalose.

Auffällig ist nun, daß bei den Patienten mit hohem Natriumgehalt der Erythrocyten sowohl die Azidose als auch die Hemmung des Natriumeinstroms im Vergleich mit den niereninsuffizienten Patienten mit normalem Natriumgehalt der Erythrocyten relativ gering war. Ein Hyperaldosteronismus, der nach den obigen Ausführungen dem Einfluß der Nierensuffizienz auf $\mathrm{pH}$ (Azidose) und $\mathrm{Na}^{+}$-Einstrom (Verlangsamung) entgegenwirken müßte, könnte dieses Verhalten erklären.

Im Gegensatz zur Natriumkonzentration war der Kaliumgehalt der Erythrocyten bei schwerer Niereninsuffizienz erniedrigt, ein Befund, wie er bereits wiederholt beschrieben wurde $(1,2,4,5)$. Die deutliche Korrelation der Kaliumkonzentration zum aktuellen $\mathrm{pH}$-Wert des Blutes weist auf die Bedeutung der Azidose für die Verminderung des intrazellulären Kaliums hin. Wie ZumKLEY und Mitarbeiter (21) zeigen konnten, besteht bei chronischer Niereninsuffizienz etwa parallel zur extrazellulären Azidose auch eine Abnahme des intrazellulären $\mathrm{pH}$-Wertes. Der intrazelluläre Anstieg an $\mathrm{H}$ Ionen macht zur Aufrechterhaltung der Elektroneutralität eine Verminderung der anderen Kationen erforderlich, die im Intrazellulärraum ja vor allem vom Kalium gestellt werden. Andererseits ist bei chronischer Niereninsuffizienz wie WELT und Mitarbeiter (3) nachwiesen, unabhängig vom extrazellulären $\mathrm{pH}$-Wert der Kaliumeinstrom (aktiver Kaliumtransport) in die Erythrocyten verlangsamt. Dieser Vorgang könnte ebenfalls die $\mathrm{Ab}$ nahme des Kaliumgehaltes der Erythrocyten erklären, vorausgesetzt, die Hemmung des Kaliuminfluxes überwiegt die durch die Azidose hervorgerufene Verlangsamung des Kaliumausstroms (11). Untersuchungen von PANKOW und Mitarbeitern (22) sprechen dafür, daß möglicherweise auch der hohe Harnstoffspiegel bei schwerer Niereninsuffizienz für die Abnahme des intrazellulären Kaliumgehaltes von Bedeutung ist.

\section{Literatur}

1. Burk, H. C. und M.D. Bundschur, 5. Symp. über aktuelle Probleme der Dialysererfahren. Innsbruck, 1969. - 2. GessLER, U., Verh. dtsch. Ges. inn. Med. 66, 870 (1960). — 3. WELT, L. G.,
J. R. Sachs und T. J. McManus, Transact. Ass. Amer. Physicians 74, 169 (1964). - 4. ZuMKLEY, H. und H. Losse, Gastroenterologia, Basel 104, 136 (1965). - 5. ZumkLEY, H., H. Losse, F. Wes- 
SELS und S. Westerboer, Verh. dtsch. Ges. inn. Med. 72, 624 (1966). - 6. Heintz, R. und D. Renner, Klin. Wscht. 43, 1167 (1965). - 7. Morgan, J. und R. E. Morgan, Metabolism 13, 629 (1964). - 8. Bolte, H.D., H. MENNINGER und G. RreCKer, Klin. Wschr. 44, 337 (1966). - 9. Solomon, A. K., J. Gen. Physiol. 36, 57 (1952/53). - 10. WesseLs, F., G. Junge-HüLSING und H. Losse, Z. Kreislaufforschg. 56, 374 (1967). - 11. Prleger, K., W. Rummel und E. Serfen, Pfïgers Arch. Physiol. 271, 378 (1967). - 12. VILlamir, M. F., V. Rettor und CH. R. KLeEMaN, J. Laborat. Clin. Med., S. Louis 72, 308 (1968). - 13. Post, R. L. und P. C. Jolıx, Biochim. biophysica Acta, Amsterdam 25,
118 (1957). - 14. Renner, D. und R. Heintz, Klin. Wschr. 44, 1204 (1966). - 15. Allison, A. C. und G. P. Burn, Brit. J. Hemat. 1, 291 (1955). - 16. Merz, D. P. und H. Sarre, Klin. Wschr. 46, 1073 (1968). - 17. LOMMER, D., G. DüsterdieCK, P. VeCser und H.P. WolfF, Klin. Wschr. 46, 741 (1968). 18. KoczoreK, KH. R., J. KARL, G. RiECKeR, M. EICKE und H. P. Wolpr, Dtsch. Med. Wschr. 84, 1134 (1959). - 19. WesSELS, F., Inaug. Dissertation Münster (1964). - 20. Wessels, F., Ges. Nuclearmed. 6. Jahrestagung (1968) (im Druck). - 21. ZuMkLeY, H. und E. Schürmeyer, diese Z. 6, 350 (1968). - 22. PaNkow, D. und K. PoHLE, diese Z. 6, 369 (1968).

Dr. F. Wessels

44 Münster (Westf.)

Westring 3 